\begin{tabular}{|c|c|}
\hline 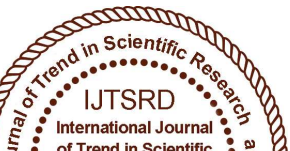 & $\begin{array}{l}\text { International Journal of Trend in Scientific } \\
\text { Research and Development (IJTSRD) }\end{array}$ \\
\hline 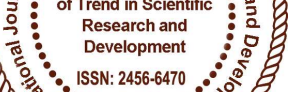 & International Open Access Journal \\
\hline 000 & ISSN No: 2456 - 6470 | www.ijtsrd.com | Volume - 2 | Issue -5 \\
\hline
\end{tabular}

\title{
An Improved DEEHC to Extend Lifetime of WSN
}

\author{
Pragati Dikshit $^{1}$, Rajveer Kaur Hyher ${ }^{2}$, Goldendeep Kaur ${ }^{2}$ \\ ${ }^{1}$ Student, ${ }^{2}$ Professor \\ CSE Department, Guru Nanak Dev Engineering College, \\ Ludhiana Punjab, India
}

\begin{abstract}
Sensor networks consist of randomly deployed nodes that are powered by smaller batteries. The quantum and quality of information sensed by these nodes is of vital importance. Battery saving techniques or algorithms are need of the hour. One such technique is clustering of the nodes. This paper presents modification to existing DEEHC algorithm that builds multiple vertex disjoint paths from nodes to base station via cluster heads. The proposed modification (MDEEHC) improves the performance of the network as equated to DEEHC and FLDEEC by relaying the data of the child cluster heads using relay nodes. MDEEHC shows improvement in terms of throughput, packet delivery ratio, number of packet drops and remaining energy.
\end{abstract}

Keywords: Sensors, disjoint paths, throughput, clustering, MDEEHC, FLDEEHC, DEEHC

\section{INTRODUCTION}

The most challenging aspect of WSN is that they are energy resource-constrained and that energy cannot be replenished. The problem arises when all the sensor nodes are required to forward the data packets to the sink node. In this process, the available energy in each node can be wasted through idle listening and retransmitting due to collisions as well as overhearing. Cluster-based WSN routing protocols excel network topology management and energy minimization. Clustering methods in WSN lead the sensor nodes to be organized into small disjoint groups, where each cluster has a coordinator referred as $\mathrm{CH}$. In cluster based approaches the sensors do not need to communicate directly with BS. Instead, the $\mathrm{CHs}$ are responsible to organize cluster members $(\mathrm{CMs})$ and send the data collected within the cluster to the BS.
This process lead to a significant reduction in the amount of transferred data in the network. Consequently, overheads in communication as well as energy consumption in clustering will be reduced significantly.

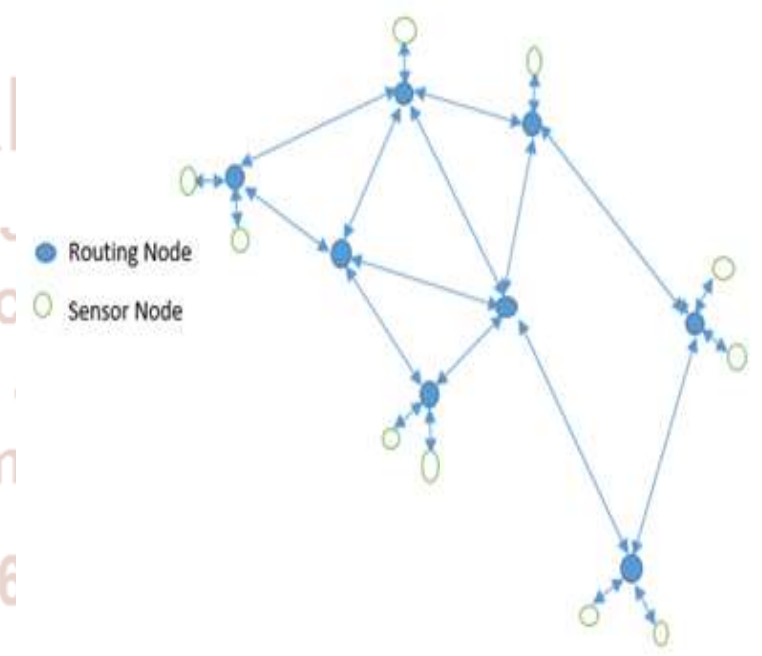

Fig.1 WSN Architecture

Maintaining the created clusters is the main challenging task in the methods. To choose a node as a $\mathrm{CH}$, it is necessary to define its eligibility. That is calculated based on local information of the nodes' current situations many of the clustering techniques talk about $\mathrm{CH}$ advertisement process in which every eligible node will broadcast an advertisement message for the election of $\mathrm{CH}$. This process will consume large amount of energy, as so many nodes will be involved in the broadcasting process. In addition, multi-hop communication approaches followed requires parent cluster heads to forward the data of child $\mathrm{CH}$ as well. This increases load over the cluster heads. This paper proposes modification to existing DEEHC by adding the concept of choosing optimal 
relay nodes to forward data from child cluster heads to base station. The remainder section of this paper is organized as follows: Section II of the paper surveys the related work, Section III depicts the research methodology, and Section IV portrays the outcomes we got from the work. Section V describes the conclusion and future work.

\section{Literature Review}

Numerous plans have just been proposed with respect to adaptation to non-critical failure in WSN to accomplish unwavering quality, unpredictability, vitality sparing and so forth. There are a portion of the papers discussed here which have talked about adaptation to internal failure methods.

Prasenjit Chanak et. al, [1], reflected the difficulty of enduring system failures realized by positioned sensors in a sensor network. They initially suggested a new clustering procedure for sensor networks, named DEEHC that chooses $\mathrm{CHs}$ conferring to the remaining energy of positioned nodes with the help of a subordinate timer. For the duration of the clustering stage, individual node discovers $k$-vertex separate routes to $\mathrm{CHs}$ reliant on the energy level of its neighbor nodes. They also suggest a kVDPR procedure where respective $\mathrm{CH}$ discovers $k$-vertex separate routes to the $\mathrm{BS}$ and communicates their amassed information to the BS. Additionally, they furthermore suggest a new RMM that can restore $k$ vertex separate routes through the observing period. The subsequent network turns out to be tolerant to $k-1$ disasters in the nastiest situation. The suggested system has been comprehensively verified by means of numerous network situations and associated to the prevailing methodologies to demonstrate its efficiency.

Sushruta Mishra et. al., [2] tried to cover issues identified with fault recognition and recovery systems in wireless sensor networks in both hypothetical and application situated research. Reliability was an essential issue in a network and when the network is energy constrained as in a wireless network then it turns into a high need to guarantee that this network is profoundly dependable. Be that as it may, this reliability is seriously influenced by mistakes and faults that happen because of different reasons, for example, Hardware failing, bugs in programming, natural dangers and so forth. Accordingly, a sensor network ought to be fault safe so it can manage these incorrect conditions adequately.
Prasenjit et. al., [3] proposed an energy productive node fault determination and recovery for wireless sensor networks alluded as fault tolerant multipath routing plan for energy effective wireless sensor network (FTMRS). The FTMRS depends on multipath information routing plan. One most limited way is use for principle information routing in FTMRS method and other two backup ways are utilized as option way for faulty network and to deal with the over-burden activity on primary channel. Most limited way information routing guarantees energy productive information routing. The execution examination of FTMRS shows better outcomes contrasted with other well-known fault tolerant methods in wireless sensor networks.

Samira et. al., [4] proposed characterization in view of the network measure, since the execution of the larger part systems relies on upon the size regarding geographical area and number of nodes. Fault tolerance is a standout amongst the most vital wireless sensor networks requirements. It guarantees that the network keeps on working accurately even when a few components fail. Truth be told, fault tolerance is a need in this sort of networks because of sensor node attributes, radio correspondences and threatening conditions in which these networks are deployed. In this study, they give a diagram of WSN components that give or enhance the fault tolerance property of wireless sensor networks.

Savita et. al., [5] displayed a basic investigation of different fault tolerance components in wireless sensor networks, for example, excess based instruments, clustering based systems and arrangement based systems to recognize the qualities and shortcomings of every one of these components. At long last, the paper presents conclusion and recommends some future research headings that will be useful for specialists who are working in this field. Fault tolerance is one of the basic issues in Wireless Sensor Network (WSN) applications. The issue of missing sensor node, correspondence connection and information are inescapable in wireless sensor networks. WSNs encounter disappointment issues because of different components, for example, control exhaustion, ecological effect, radio obstruction, disengagement of sensor node and impact. Numerous specialists have proposed fault tolerant instruments that can accomplish higher information reliability, precision, energy saving, improve network lifetime 
and limit disappointment of segments of wireless sensor network.

Doo soon park [6] proposed a measure to distinguish and manage sensors by partitioning WSN into settled WSN and portable WSN. This paper additionally proposes a strategy to predict and express the energy utilization of a sensor network. The commercialization of recognition and observation frameworks by sensor networks has as of late turned out to be more prominent in different ventures and in common life. Wireless sensor networks (WSNs) might be used in territories. WSNs, which have three essential components, ought to have the capacity to convey through a blend of network and sensor capacities. In expansive scale sensor networks, however, support expenses to replace nodes when they are out of request, and batteries utilized for wireless interchanges, extraordinarily increment, notwithstanding the cost of building them. In this way, measures to distinguish and react to sensor faults in a sensor network, and additionally an instrument to predict the energy utilization of sensors, are essential.

Cho H., et. al., [7] proposed Construction algorithms for reliable CDS (CARCODS). This is the technique by which the execution of specially appointed topologies that comprise of CDSs is made strides. A CDS arrangement technique was recommended by making utilization of the telecom time deferral of the neighboring setup notice message that components in the rest of the battery limit, the portability of nodes, and the quantity of neighboring nodes.

Hong Y. et. al., [8] proposed partial reconstruction of CDS (PRCDS). A CDS fractional reconfiguration algorithm was proposed to adequately respond to issues because of the event of basic nodes when a CDS-based routing convention was connected. In designing the CDS nodes for load adjusting, this strategy scans for the likelihood of association, while topology reconfiguration, because of basic nodes, is expanded into two-hope nodes. Subsequently, the nodes inside a particular range in terms of their reconfiguration time.

Zhang, et, al [9] proposed a fuzzy Logic based distributed energy-efficient clustering algorithm (FLDEEC). During cluster head $(\mathrm{CH})$ election, it takes nodes' energy, nodes' degree and neighbor nodes' residual energy into consideration as input parameters. Through fuzzy interference system, node uses its own information and its neighbor nodes' information to make a judgment to decide whether it fits to be a $\mathrm{CH}$ or not in a distributed way. FLDEEC algorithm can solve hot spot problem caused by communication in cluster, improve the efficiency of data transmission and extend the lifetime of the whole network by screening out nodes with bad conditions. The experimental results indicated that the FLDEEC algorithm is better than CHEF algorithm and DEEC algorithm in the aspects of data transmission, energy consumption and the number of living nodes.

\section{Research Methodology:}

The proposed scheme works in the following manner:

\section{Modified DEEHC algorithm}

Initially each sensor node will check its current energy level. If the current energy level is more than the threshold value, then it starts advertisement process where it broadcasts advertisement message to all the sensors in its communication range. The message contains its ID, location information and its residual energy. During this process, if any other node, which has received the advertisement packet, checks that its current energy is less than received energy value in the advertisement packet then it withdraws itself from advertisement process and becomes cluster member. If any node receives multiple advertisement messages, then it would join the cluster head for which energy is highest. When the non- $\mathrm{CH}$ node broadcasts a join packet to the $\mathrm{CH}$ to join its cluster, it would additionally broadcast its location information and residual energy as well. The current $\mathrm{CH}$ would therefore collect all the data from its cluster members. This data will be used to rotate the $\mathrm{CHs}$ for the subsequent rounds. The current $\mathrm{CH}$ will sort the members in the order of highest energy and minimum distance from the base station. The member at the top of the list would be selected as $\mathrm{CH}$ for the subsequent round. Thus, at the beginning of every round, the $\mathrm{CH}$ advertisement phase would be avoided.

\section{Modified k-vertex disjoint paths routing}

Once the clusters have been formed and nodes have been arranged in the order of becoming $\mathrm{CHs}$ for the subsequent rounds, the base station will begin broadcasting of L-ADVE to the cluster heads in its communication range. The output of the existing scheme would be multiple disjoint paths from $\mathrm{CHs}$ to the BS. Once the various paths have been formulated, the every $\mathrm{CH}$ would select a member node from its cluster to relay the data of the child $\mathrm{CH}$. The member node would be the one having highest residual energy 
and least distance from the midpoint of child $\mathrm{CH}$ and BS. Thus, every $\mathrm{CH}$ would now relay the data to the chosen relay node which would act as parent to child $\mathrm{CH}$. This would reduce the load over the $\mathrm{CH}$ to relay the data of other CHs as well.

\section{Modified Route Maintenance Mechanism}

In this mechanism, the focus will be given on the disjoint path made between the $\mathrm{CHs}$ and $\mathrm{BS}$ via chosen relay nodes. If residual energy of any chosen relay node falls to less than 80 percent of the initial energy value or any node goes faulty then the next cluster member will act as relay node for the child $\mathrm{CH}$. This would further lead to better routes formed in the network.

\section{Algorithm of the modified DEEHC}

1. Suppose ' $N$ ' is the number of the nodes in the network

2. For $i=1: N$

A. If Remaining Energy(i) > Threshold

I. Broadcast ADV packet in the communication range

B. Else

I. Do not opt for broadcasting

C. End if

D. If Remaining energy $<$ Energy received in the ADV packet

I. Withdraw from advertisement

E. Else

I. Continue with broadcasting process

F. End if

3. End for

4. Suppose ' $M$ ' be the number of nodes which received ADV packet

5. For $\mathrm{i}=1: \mathrm{M}$

A. If ADV packet is received from multiple $\mathrm{CHs}$

I. Form k-vertex paths with them

II. Choose highest energy $\mathrm{CH}$ as parent $\mathrm{CH}$

B. Else

I. Keep cluster head in the parent $\mathrm{CH}$ list

C. End if

6. Form cluster with the selected parent $\mathrm{CH}$

7. Send Join packet to the $\mathrm{CH}$
8. End for

9. For each $\mathrm{CH}$, do

A. Arrange members in increasing order of residual energy and least distance from BS

B. Choose node at top of the list to be $\mathrm{CH}$ for the next round

10. End for

11. Base station broadcasts L-ADVE packets to $\mathrm{CH}$

12. Each $\mathrm{CH}$ re-broadcasts L-ADVE packet to child $\mathrm{CHs}$

13. Suppose ' $k$ ' is the number of disjoint paths from $\mathrm{CH}$ to $\mathrm{BS}$

14. For $\mathrm{i}=1: \mathrm{k}$

A. Each $\mathrm{CH}$ chooses relay node from its member nodes

15. End for

16. If any node goes fault

A. $\mathrm{CH}$ chooses next member as relay node for child $\mathrm{CH}$

End if

\section{Implementation \& Results}

In this work, three techniques have been simulated namely FLDEEC [9], DEEHC [1] and third was modification to DEEHC, which we named ModifiedDEEHC or MDEEHC. All the three techniques are simulated in Network Simulator 2.35. The following set of input parameters were considered while creating a wireless sensor network:

\begin{tabular}{|c|c|}
\hline Parameter & Value \\
\hline Channel & Wireless \\
\hline Mac & 802.11 \\
\hline Propagation Model & Two Ray Ground \\
\hline Antenna & Omni Directional \\
\hline Number of nodes & 100 \\
\hline Network Area & $700 * 750$ sq meters \\
\hline Initial Energy & 20 Joules \\
\hline Queue & CMUPriqueue \\
\hline Queue Length & $\mathbf{5 0 0}$ \\
\hline
\end{tabular}

Table 4.1: Simulation Parameters

The performance of the network was measured based on throughput, packet delivery ratio, number of packet drops and energy remaining in the network. 


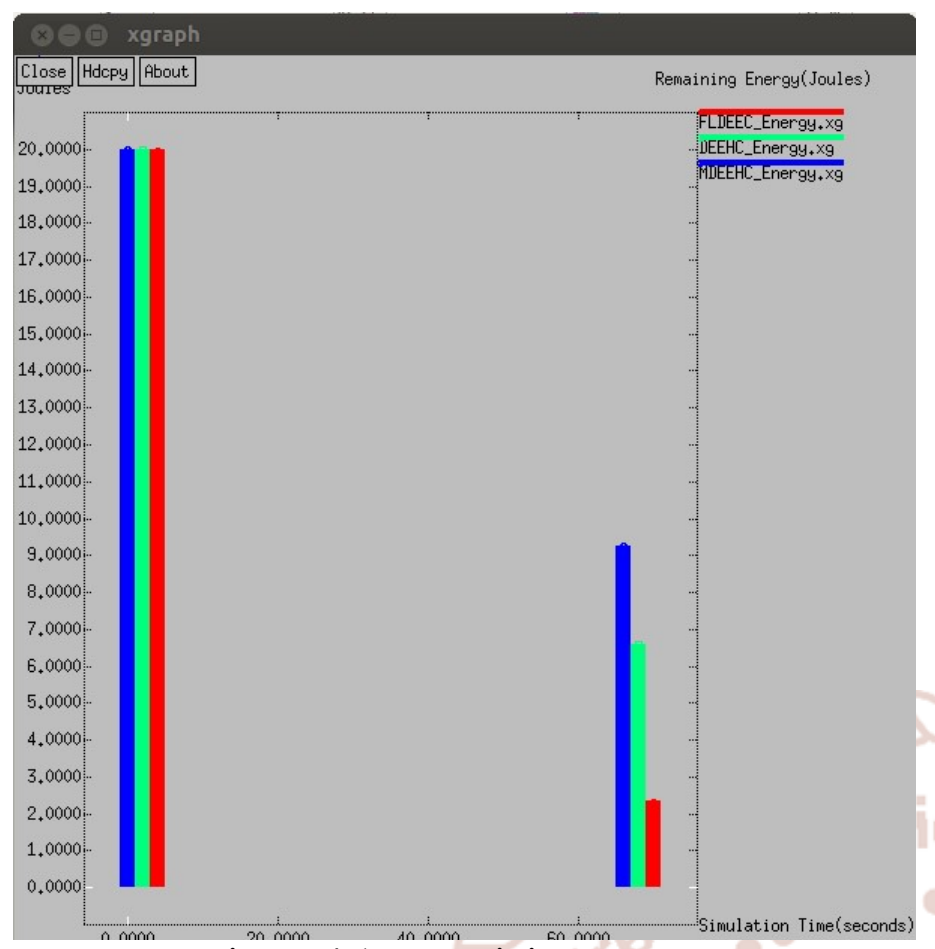

Figure 4.1: Remaining energy

The initial average energy of the network was 20 Joules. At the end of simulation, the remaining energy was approx. 9 Joules for M-DEEHC, 6.5 Joules for DEEHC and 2 Joules for FLDEEC. The amount of remaining energy is highest for proposed technique. Initially, all the three schemes opt for cluster formation process. This process requires substantial amount of broadcasting leading to consumption of energy sources of the sensors. This process is run every time at the start of new round for the cluster head selection process. The proposed scheme, however, avoids successive runs of this process by electing the cluster heads for the subsequent rounds in the current round itself. Thus, nodes do not need to advertise themselves for the cluster head election process. The proposed scheme proposes that cluster heads do not take on the load of forwarding the data of the child cluster heads. Instead, the parent cluster heads choose a relay node from their clusters to forward the data from child cluster head to the base station. Thus, these two reasons when combined, leads to lesser energy consumption of the network as well as increased lifetime.

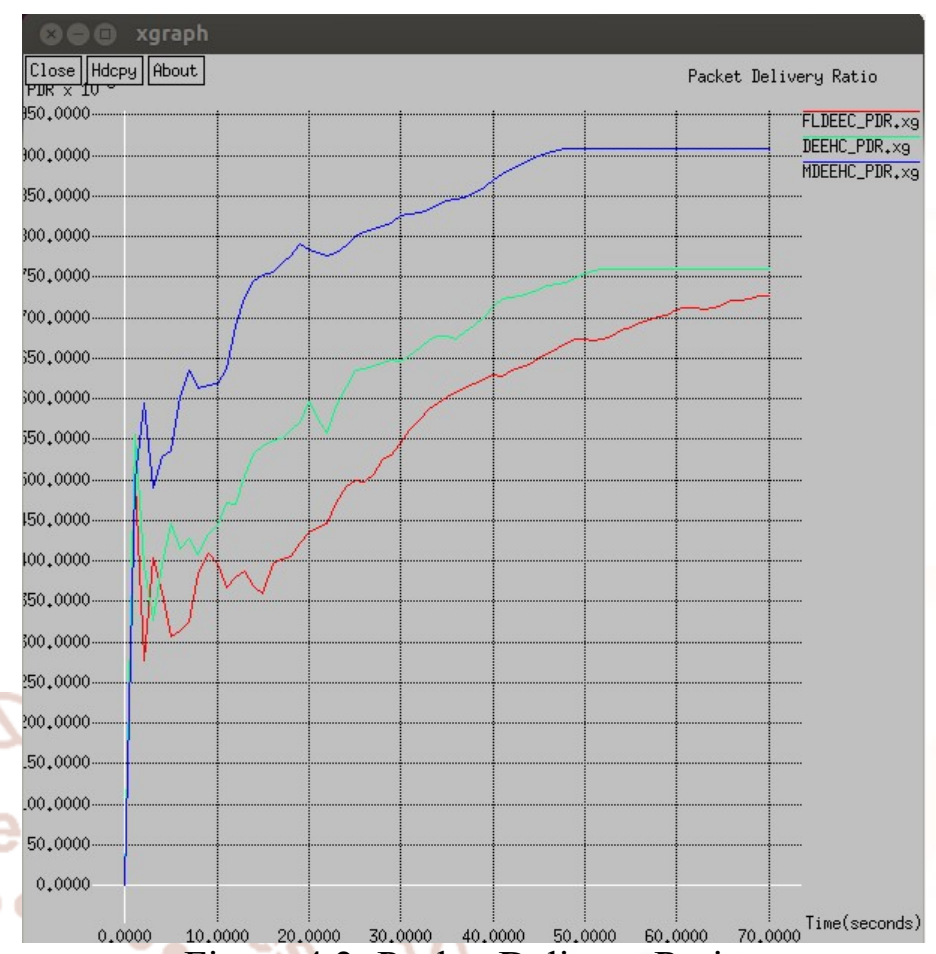

Figure 4.2: Packet Delivery Ratio

In M-DEEHC, the cluster heads for the subsequent rounds are selected in the previous round which avoids broadcasting of ADV packets in consecutive rounds. Thus, the least amount of broadcasting results in least congestion and best value for packet delivery ratio for M-DEEHC. Consequently, after the cluster formation, value of PDR increases during data packet transmission in the network. The value for PDR is 0.91 for M-DEEHC, 0.76 for DEEHC and 0.72 for FLDEEC.

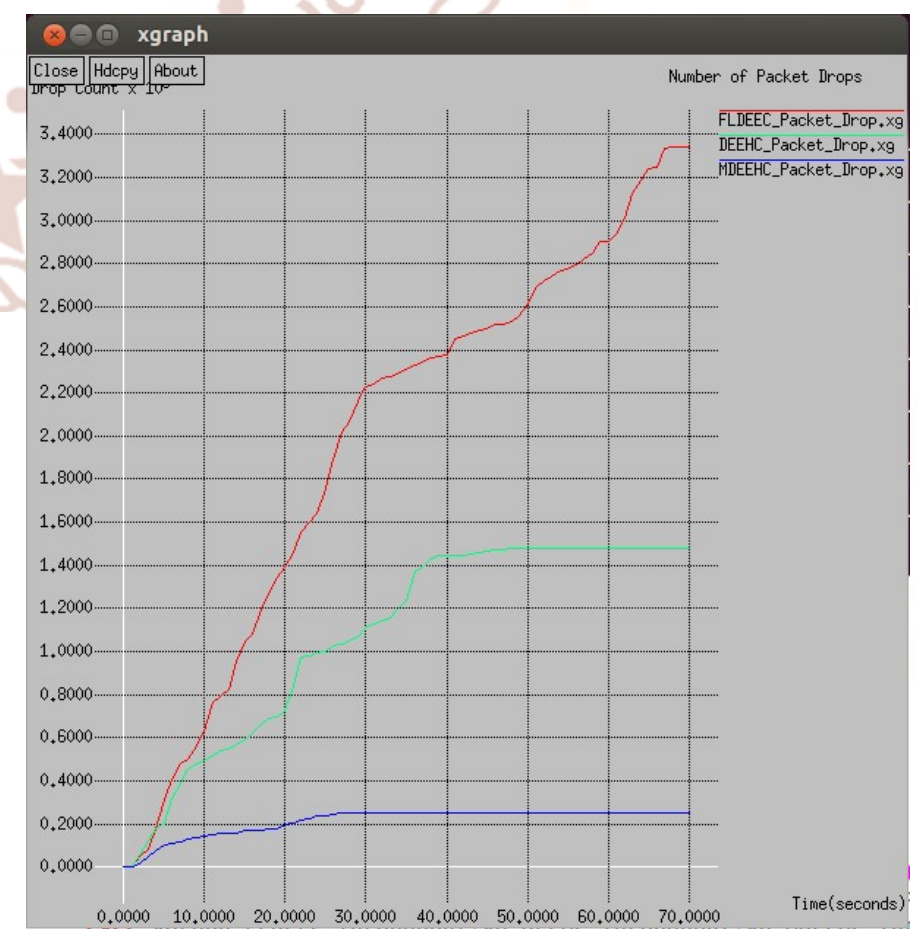

Figure 4.3 Number of Packet Drops 
It can be seen from the graph that our proposed scheme shows very less number of packet drops in the network. This is because of reduced congestion (by prevention of multiple times broadcasting of ADV packets) in the network. The number of packet drops were recorded highest for FLDEEC scheme and lesser for DEEHC.

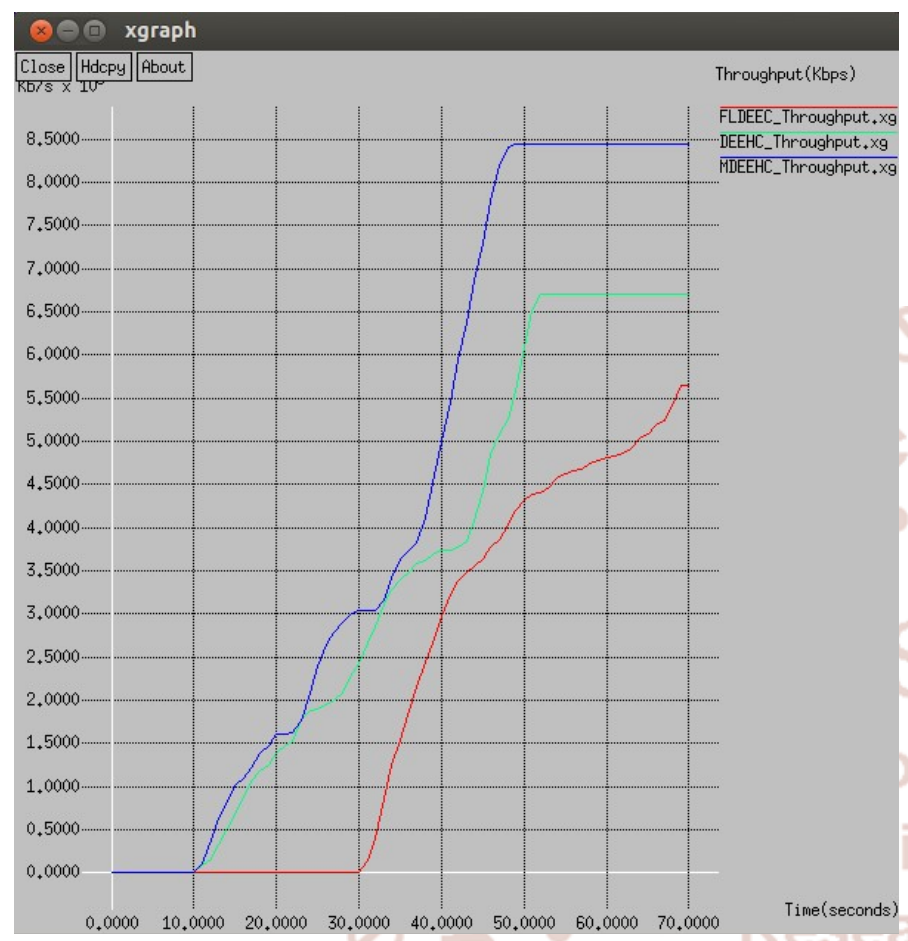

Figure 4.4: Throughput remaining energy, number of packet drops and packet delivery ratio. The initial average energy of the network was 20 Joules. At the end of simulation, the remaining energy was approx. 9 Joules for MDEEHC, 6.5 Joules for DEEHC and 2 Joules for FLDEEC. The amount of remaining energy is highest for proposed technique. In M-DEEHC, the cluster heads for the subsequent rounds are selected in the previous round, which avoids broadcasting of ADV packets in consecutive rounds. Thus, the least amount of broadcasting results in best value for packet delivery ratio for M-DEEHC. The value for PDR is 0.91 for M-DEEHC, 0.76 for DEEHC and 0.72 for FLDEEC. This also leads to lesser values for number of packet drops in the network. For M-DEEHC, the value of throughput was around $8500 \mathrm{Kbps}$, for $\mathrm{DEECH}$ this value was around $6800 \mathrm{Kbps}$ and for FLDEEC the value for $5700 \mathrm{Kbps}$. Thus, it can be concluded that our proposed scheme outperforms the other schemes.

In future, other parameters such as delay, jitter etc. can be analyzed for the proposed scheme. These days, the concept of Internet of Things is in high demand. This work can also be explored for an application of IoT such as military surveillance, forest fire detection etc. Internet of Things combine sensor networks along with other networks such as MANETs, VANETs or FANETs. M-DEEHC can be explored for the same.

This indicates amount of data received at the destination node per unit of time. Lesser packet drops leads to increase the throughput of the network for MDEEHC. The value of throughput is in line with the value obtained for PDR as well. For M-DEEHC, the value of throughput was around $8500 \mathrm{Kbps}$, for $\mathrm{DEECH}$ this value was around $6800 \mathrm{Kbps}$ and for FLDEEC the value for $5700 \mathrm{Kbps}$.

\section{Conclusion and Future Work}

In this work, we have implemented FLDEEC, DEEHC and modification to existing DEEHC clustering algorithm. FLDEEC defines the selection of cluster head according to fuzzy rules. DEEHC defines the formation of k-vertex disjoint paths from cluster members to cluster heads and from cluster heads to sink node or base station. This algorithm selects cluster heads considering remaining energy of the nodes as prime factor. M-DEEHC modifies the cluster formation of DEEHC algorithm as well as modifies the way the data is transmitted from child cluster heads to the base station. The performance of the network was analyzed based on throughput,

\section{References}

1. Prasenjit Chanak, Indrajit Banerjee, R. Simon Sherratt, "Energy-Aware Distributed Routing Algorithm to Tolerate Network Failure in Wireless Sensor Networks", Ad Hoc Networks, 2016.

2. Sushruta Mishra, Lambodar Jena and Aarti Pradhan," Fault Tolerance in Wireless Sensor Networks" in International Journal of Advanced Research in Computer Science and Software Engineering Oct 2012.

3. Prasenjit Chanak, Tuhina Samanta, Indrajit Banerjee, "Fault-Tolerant Multipath Routing Scheme for Energy Efficient Wireless Sensor Networks" in international Journal of Wireless \& Mobile Networks (IJWMN) Vol. 5, No. 2, April 2013.

4. Samira Choukhi," A survey on fault tolerance in small and large scale wireless sensor networks" in ELSEVIER Sep 2015. 
5. Savita Gupta and Sukhwinder singh, "A survey on fault tolerance techniques in Wireless Sensor Networks" in IEEE 2016.

6. Doo soon park, "Fault Tolerance and Energy Consumption Scheme of a Wireless Sensor Network" in international journal of distributed sensor Nov 2013.

7. Cho H. -S., Yoo S.-J, "Power, mobility and wireless channel condition aware connected dominating set construction algorithm in the wireless ad-hoc network "in the Journal of Korea Information and Communications Society 2005.

8. Hong Y. -S., Lim H. -S. A, "load-balanced topology maintenance with partial topology reconstruction" in the Journal of Korea Information and Communications Society 2010.

9. Ying Zhang, Rundong Zhou, "Networks Energy-Efficient clustering algorithm based on fuzzy interference system", 29th Chinese Control and decision policies (CCDC), IEEE, 2017.

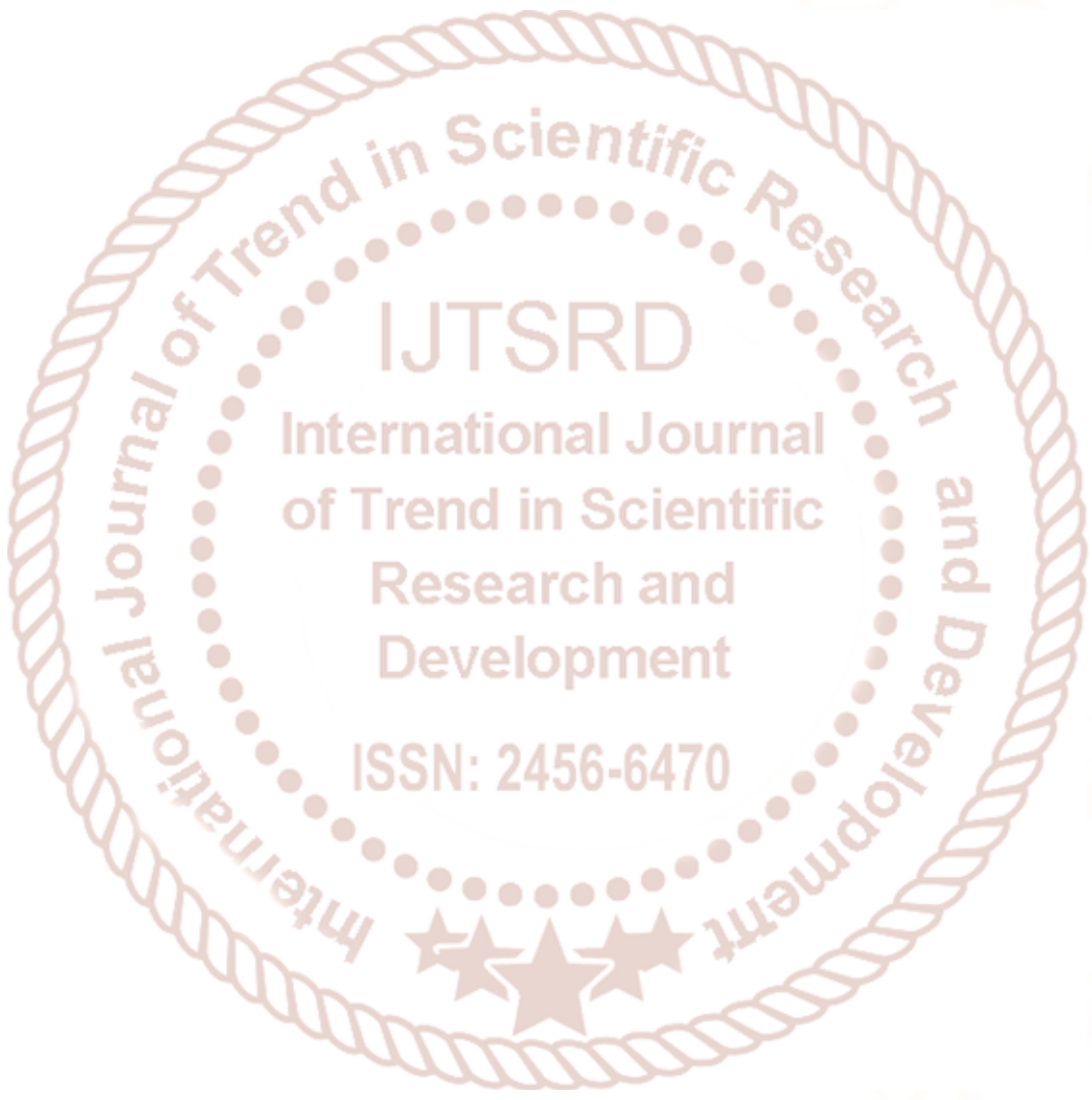

